\title{
Investigation of a significant increase in referrals during neonatal hearing screening: a comparison of Natus ALGO Portable and ALGO 3i
}

\author{
Michael Hofmann \\ Heleen Luts \\ Hanne Poelmans \\ Jan Wouters
}

ExpORL, Dept. Neurosciences, K.U.Leuven, O\&N 2, Herestraat 49 bus 721, 3000 Leuven, Belgium

\begin{abstract}
Objective: Neonatal hearing screening programs in Flanders and the Netherlands use Natus ALGO screening devices. Since 2006 in Flanders and 2009 in the Netherlands, both programs have replaced the older ALGO Portable devices with the newer ALGO 3 i devices. However, in both countries, ALGO 3i devices have a significantly higher rate of referrals than ALGO Portable devices. In Flanders, the refer rate has more than doubled with the switch to ALGO 3i devices. In the Netherlands, screening centers which used ALGO $3 \mathrm{i}$ devices also showed a significant increase in referrals. In both countries, the percentage of children diagnosed with permanent hearing loss remained approximately the same. Design: A technical comparison of both device types was carried out to identify possible causes for the increase in referrals. The stimulus output of two ALGO Portable and three ALGO 3i devices was recorded and analyzed for stimulus level, spectral properties and stimulus irregularities. Results: ALGO 3i devices stimulate at a peak level 4.6 dB lower than ALGO Portable devices, have a different stimulus spectrum and show unexplained stimulus irregularities during $4 \%$ of the stimulation time. Conclusions: A number of technical differences were found between both device types which could explain the increase in referrals.
\end{abstract}

\section{Introduction}

In most developed countries, neonatal hearing screenings take place in the first weeks after birth to detect unilateral and bilateral permanent hearing impairment of $40 \mathrm{~dB}$ or more. These screenings rely on objective measures such as Otoacoustic Emissions (OAEs), Auditory Brainstem Responses (ABRs) or Auditory Steady State Responses (ASSRs) (Joint Committee on Infant Hearing 2007).

In Flanders, a region of Belgium, a neonatal hearing screening program was implemented in 1998 that consists of two successive rounds of screening based on Automated Auditory Brainstem Responses (AABRs), followed by a referral for further clinical evaluation on positive screening outcomes in both rounds (Van Kerschaver et al. 2007). For

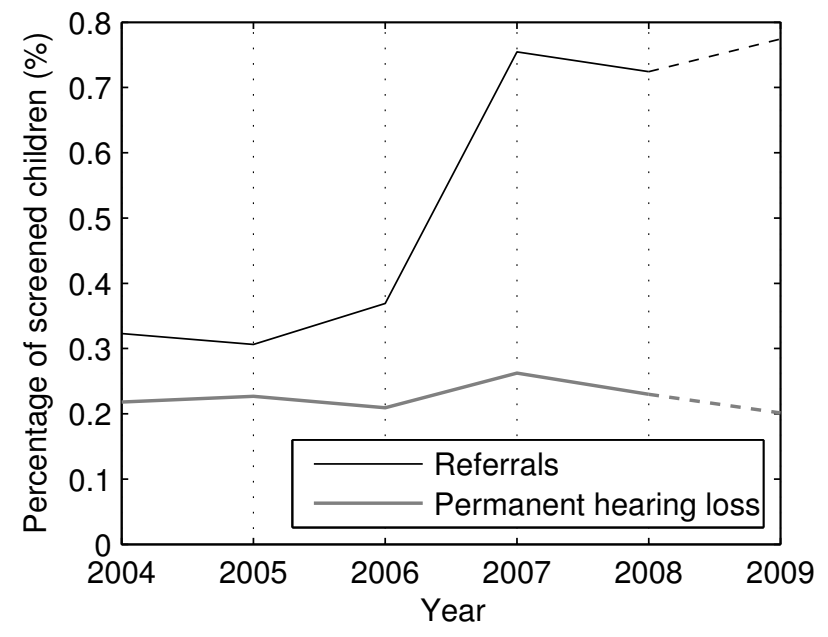

Figure 1: Neonatal hearing screening results for Flanders. Dashed lines: preliminary data. (Van Kerschaver and Stappaerts 2008)

the screening, Natus ALGO Portable devices had been used until the end of 2006 when they were superseded by Natus ALGO $3 \mathrm{i}$ devices. Other than the integration of the ALGO $3 \mathrm{i}$ devices' feature to synchronize patient screening results with a central database, no change of screening procedure or parameters took place during the switch to the new devices.

With about $2.3 \%$ of all screened children in Flanders, the percentage of permanently hearing impaired children has been more or less constant over the years (figure 1). The percentage of children referred for further clinical evaluation on the other hand has increased substantially with the switch to the new ALGO 3i devices. Between 2005 and 2007, when the switch to the new devices took place, the number of children referred has increased from $3.1 \%$ to $7.6 \%$ of all screened children and remained constant afterwards (Van Kerschaver and Stappaerts 2008). This change corresponds to a sixfold increase in false positives, mostly consisting of children with slight temporary conductive hearing impairments.

In the Netherlands, a general neonatal hearing screening 
program organized by the National Institute for Public Health and the Environment (RIVM) was introduced between 2002 and 2006. Newborns are screened in three successive rounds, and are referred for further clinical evaluation on positive screening outcomes in all three rounds. While the first two rounds use transient-evoked OAEs, the third round is also based on ABRs measured with ALGO Portable and later ALGO 3i devices.

In 2009, the program in the Netherlands was evaluated by the independent research institution Netherlands Organization for Applied Scientific Research (TNO). For children without permanent hearing loss that took part in the third round of screening, the number of referrals for the ALGO Portable devices were $6.5 \%$ and $6.7 \%$ for screening organizations that conducted the first two rounds at home or in a screening center, respectively. For screening organizations that switched to ALGO $3 \mathrm{i}$ devices, these numbers increased to $9.5 \%$ and $12.7 \%$, respectively (van der Ploeg and Rijpstra 2010, pages 19-20).

To evaluate the stimulus differences between the two used device types, a technical comparison of ALGO Portable and ALGO $3 \mathrm{i}$ devices has been worked out. The objectives of this study were to check for (1) level differences, (2) spectral differences, and (3) stimulus irregularities.

\section{Materials and methods}

Stimulation Five different neonatal hearing screening devices were tested: two ALGO Portable devices and three ALGO 3i devices.

The two ALGO Portable devices were provided by Kind $\&$ Gezin, the organization responsible for the hearing screening program in Flanders. These devices are older neonatal hearing screening devices that were used in the neonatal hearing screening program in Flanders until 2006 when Kind \& Gezin made the switch to the newer ALGO 3i devices. The devices stimulate with alternating polarity clicks at a fixed click rate of $38 \mathrm{~Hz}$ and at a specified stimulus intensity of $35 \mathrm{dBnHL}$. A proprietary EEG pattern matching algorithm is used to determine the presence of an ABR.

Kind \& Gezin also provided three ALGO 3 i devices that are currently in use in their neonatal hearing screening program. These are the most modern hand-held devices that are currently sold by Natus for neonatal hearing screening purposes. They stimulate with alternating polarity clicks at two different click rates, which changes between 34 and $37 \mathrm{~Hz}$ every $20 \mathrm{~s}$, and at a specified stimulus intensity of $35 \mathrm{dBnHL}$. These devices use an EEG pattern matching algorithm similar to that in the ALGO Portable devices.

Recording Recordings were done in a sound booth, with a measurement setup equivalent to that required by the Natus ALGO 3i calibration procedure. Transducers were connected to the ear caps which were placed on a flat plate adapter (plexiglas, $70 \times 70 \times 10 \mathrm{~mm}$, part of the Natus acoustic check kit) on a B\&K 4152 artificial ear (figure 2). ALGO Portable and ALGO $3 i$ devices use different ear caps and positions of the transducers relative to the ear caps. While the transducers of ALGO Portable devices connect to the short side of Natus ALGO Portable ear couplers, the transducers of ALGO $3 \mathrm{i}$ devices connect to the long side of Natus Valu-Pak earphones (figure 2). The artificial ear was connected to a sound level meter B\&K 2260, which was used for all measurements in this report. Stimulus level was determined in $\operatorname{dBSPL}(\mathrm{A})$ as that was the measure used in the Natus calibration instructions for the ALGO $3 \mathrm{i}$ devices in the Natus acoustic check kit. Additionally, measurements were carried out in dBpeSPL(A) to account for the pulsatile character of stimulation and to allow for the comparison of stimulus level across device types as click rates were different for ALGO Portable and ALGO 3 i devices.

For time-domain and spectral analysis, the sound level meter was connected to a measuring amplifier B\&K 2610 with a $22.4 \mathrm{~Hz}$ high-pass which in turn was connected to an RME Multiface II soundcard, with inputs and outputs at $-10 \mathrm{dBV}$. Recordings were done on a laptop with Adobe Audition at $96 \mathrm{kHz}$ sample rate and 24 bit sample width. The stimulus waveforms for both transducers on all devices were recorded for one minute. Additionally, a recording of a B\&K 4230 $94 \mathrm{dBSPL}$ sound level calibrator was made to calibrate the recording setup. All recordings were then analyzed in MATLAB. For each recorded waveform, the individual clicks were detected, their polarity was determined by the polarity of the initial peak, they were sorted by polarity, inverted if necessary, averaged and their power spectral density was calculated.

\section{Results}

Level differences Stimulus levels of the ALGO Portable and ALGO 3i devices were compared. Mean stimulus levels were 59.2 dBSPL $(\mathrm{A})(\mathrm{SD}=0.5 \mathrm{~dB})$ and $55.6 \mathrm{dBSPL}(\mathrm{A})$ $(\mathrm{SD}=0.3 \mathrm{~dB})$ for the ALGO Portable and ALGO $3 \mathrm{i}$ devices, respectively. For the amplitude of the stimulus clicks, mean peak levels were 79.6 dBpeSPL $(\mathrm{A})(\mathrm{SD}=0.6 \mathrm{~dB})$ and $75.0 \mathrm{dBpeSPL}(\mathrm{A})(\mathrm{SD}=0.2 \mathrm{~dB})$ for the ALGO Portable and ALGO 3i devices, respectively.

Spectral differences Click shapes and power spectral densities of the ALGO Portable and the ALGO 3i devices were compared (figure 3). Power spectral densities were similar for both device types up to $4 \mathrm{kHz}$, but differed for higher frequencies. In comparison to the clicks of the ALGO Portable devices where a broad energy peak occurred between 4.5 to $8.5 \mathrm{kHz}$, the clicks of the ALGO $3 \mathrm{i}$ devices exhibited a narrower high-frequency peak at about 7 to $9 \mathrm{kHz}$.

Stimulus irregularities The recorded stimulus waveforms were visually inspected for any irregularities and analyzed regarding click rate and polarity. While the stimulus waveforms of the ALGO Portable devices did not show deviations from an alternating click train at $38 \mathrm{~Hz}$, the recordings of the ALGO 3i devices showed stimulus irregularities. The ALGO $3 \mathrm{i}$ devices use alternating polarity click trains that 

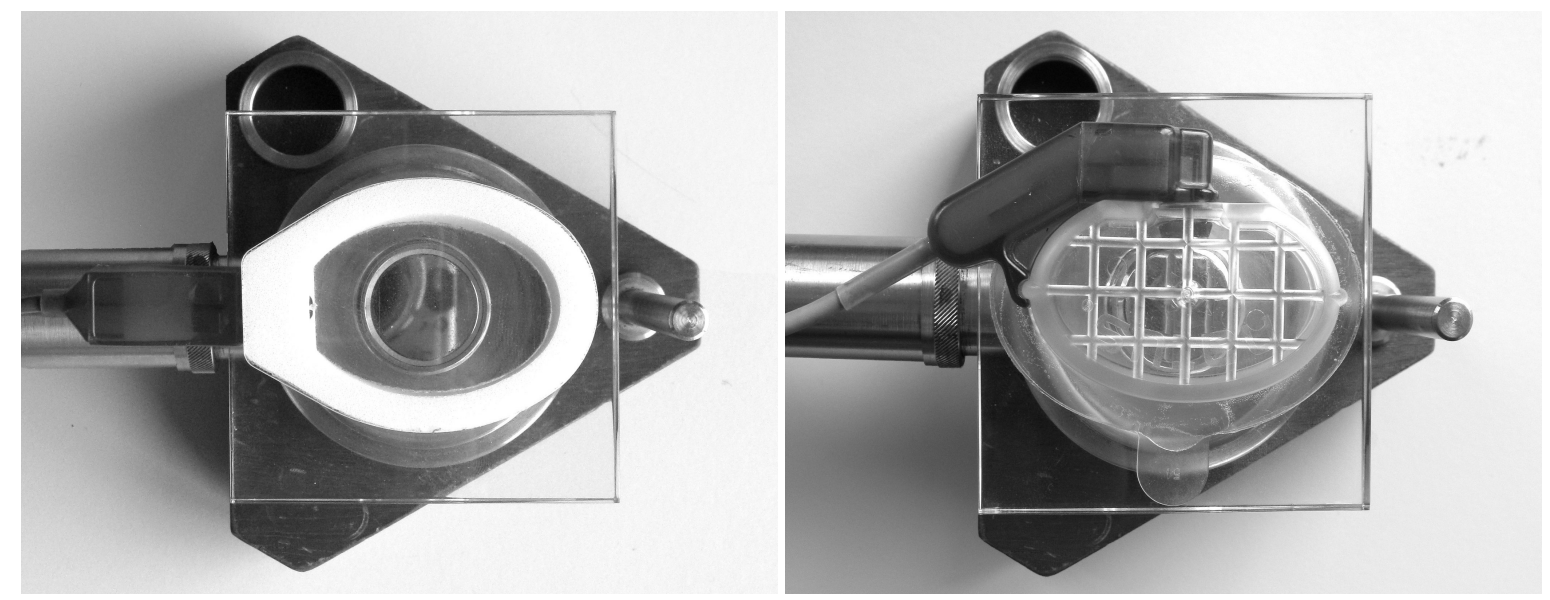

Figure 2: Recording setup as used for the ALGO Portable (left) and ALGO 3i (right) devices. ALGO transducers connected to ear caps placed on the flat plate adapter mounted on the B\&K 4152 artificial ear.

change click rate every $20 \mathrm{~s}$, and for all devices checked, each of these click rate changes happened in an irregular way. Each change was followed by three groups of stimulation irregularities consisting of (1) shifted clicks, resulting in distances between successive clicks that were neither corresponding to 34 and $37 \mathrm{~Hz}$, and (2) click polarity inversions, where clicks were of a different polarity than what would have been expected from an alternating pattern. An example can be seen in figure 4. Across all ALGO 3i devices, these irregularities were present during $0.77 \mathrm{~s}$ after each click rate change, or approximately $4 \%$ of the stimulation time.

\section{Discussion}

Level differences As all of the tested devices had been calibrated by Natus, the significant difference of $4.6 \mathrm{~dB}$ between the stimulus peak level of the ALGO Portable and ALGO 3i devices can only be explained by a different calibration procedure of the manufacturer for the different device types. The decrease in stimulus peak level with the ALGO $3 \mathrm{i}$ devices compared to the ALGO Portable devices would result in an increased sensitivity, as evoked potentials would show reduced amplitudes, increased latencies and different overall response shapes. Next to the anticipated permanent hearing impairments, this increased sensitivity would also result in the additional detection of mild temporary conductive hearing impairments as observed in Flanders (Van Kerschaver and Stappaerts 2008).

Spectral differences The differences in click shape and spectrum could be explained by the different types of transducers used by the ALGO Portable and ALGO 3i devices. Besides the type of the transducer, the different position of the transducers relative to the ear cap could also be a cause of the observed shape and spectrum differences. The differences in spectrum hint at a different excitation pattern inside the cochlea. Hearing threshold levels determined with ABRs correlate mainly with a stimulus frequency range of 2 to $4 \mathrm{kHz}$ (Gorga and Neely 2002), but ABRs near threshold can also be evoked by frequencies as low as $400 \mathrm{~Hz}$ (Don and Eggermont 1978). As spectral differences are minimal in this region, only limited effects on the sensitivity of the screening are expected.

Stimulus irregularities The stimulus irregularities seen during the click rate changes with the ALGO $3 \mathrm{i}$ devices result in clicks with shifted positions and inverted polarities. It is unknown to the authors whether the averaging in the ABR recording and analysis system in the ALGO $3 \mathrm{i}$ devices is synchronized with the irregular click positions or whether the theoretical click positions are used. In the latter case, the averaging of approximately $4 \%$ of the responses is not synchronized with the stimulation, which would lead to an increase in noise and therefore measurement time. As some of the irregularities are stable over time, the averaging would also result in a wrong response shape and would therefore affect test sensitivity.

\section{Conclusions}

When compared to ALGO Portable devices, ALGO 3i devices exhibit multiple differences with regards to stimulus characteristics and presentation: (1) different stimulus peak levels of ALGO Portable and ALGO 3i devices, (2) different stimulus waveforms caused by different transducers and transducer positions, and (3) stimulus irregularities with ALGO 3i devices.

As there is no data available that shows that the difference in stimulus level, click rate and transducers between ALGO Portable and ALGO 3i devices and the stimulus irregularities during the transition between the different click rates in ALGO $3 \mathrm{i}$ devices have no influence on the screening results in neonates, a study directly comparing the performance of both devices types in neonates is necessary.

In our opinion, the technical differences found between ALGO Portable and ALGO 3i devices could explain the in- 

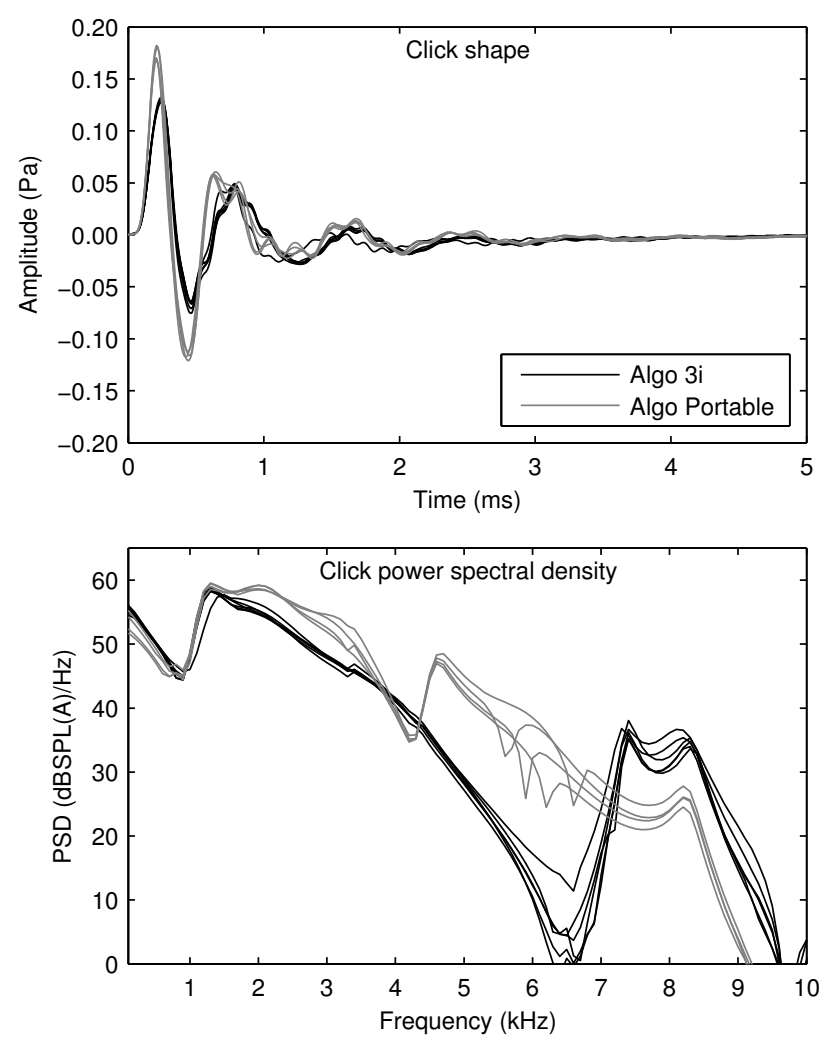

Figure 3: Click characteristics for the ALGO Portable (two devices with in total four transducers) and the ALGO $3 \mathrm{i}$ devices (three devices with in total six transducers).

crease in referrals seen in both Flanders and the Netherlands. As the number of children referred for further clinical evaluation is crucial for the costs of a neonatal hearing screening program, screening organization should put more emphasis on the evaluation of the false positive rate of screening devices, e. g. by comparing the results from multiple different device types during pilot studies, even if the devices use the same underlying physiological measurement such as ABRs or OAEs.

\section{Acknowledgments}

Portions of this research have been presented by Erwin Van Kerschaver at the Conference on Neonatal Hearing Screening, Como Lake, Italy, June 8-10, 2010. We would like to thank Luc Stappaerts and Erwin Van Kerschaver of Kind \& Gezin for bringing this issue to our attention and for providing us with ALGO devices. We also thank Anneke MeuweseJongejeugd from the RIVM for pointing out the results of the hearing screening program in the Netherlands. We acknowledge Tessa Akkerman, Christoph van der Reyden and Dirk van Hek from Cordial Medical for providing us with ALGO devices and additional measurement equipment.

A technical report detailing these findings and a list of technical and scientific questions was communicated to $\mathrm{Na}$ -

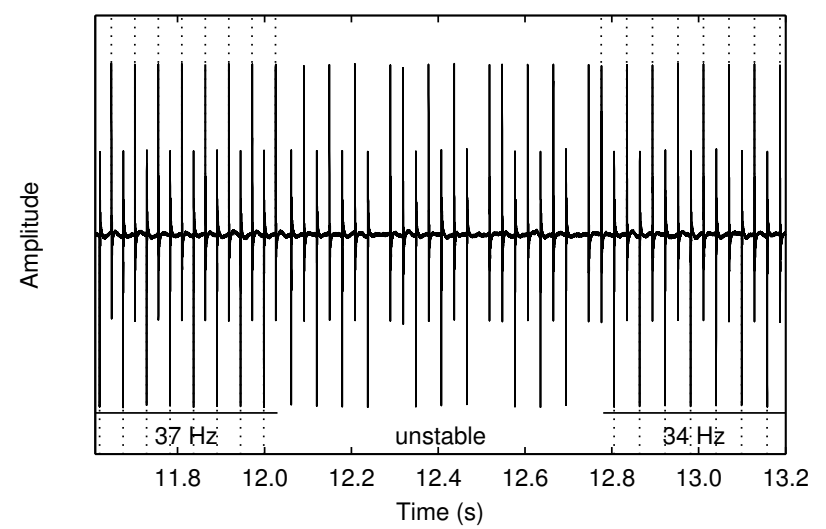

Figure 4: Example for the stimulation irregularities of the ALGO 3i devices. Stimulation waveform, zoomed into a transition from 37 to $34 \mathrm{~Hz}$; dotted lines: regular click positions and polarities.

tus in March 2010. This initial contact and further repeated requests for urgent investigation have not resulted in any concrete communication yet.

\section{References}

M. Don and J. J. Eggermont. Analysis of the click-evoked brainstem potentials in man using high-pass noise masking. J Acoust Soc Am, 63(4):1084-92, Apr. 1978.

M. P. Gorga and S. T. Neely. Some Factors that May Influence the Accuracy of Auditory Brainstem Response Estimates of Hearing Loss. In R. C. Seewald and J. S. Gravel, editors, A Sound Foundation Through Early Amplification 2001, Proceedings of the Second International Conference, pages 49-61. Phonak AG, Chicago, 2002.

Joint Committee on Infant Hearing. Year 2007 position statement: Principles and guidelines for early hearing detection and intervention programs. Pediatrics, 120(4): 898-921, Oct. 2007.

C. P. B. van der Ploeg and A. Rijpstra. Monitoring van de neonatale gehoorscreening door de jeugdgezondheidszorg in 2009. TNO Kwaliteit van Leven, Leiden, the Netherlands, 2010.

E. Van Kerschaver and L. Stappaerts. Jaarrapporten Gehoor 2004-2008 and personal communication. Kind en Gezin, Brussels, Belgium, 2008.

E. Van Kerschaver, A. N. Boudewyns, L. Stappaerts, F. L. Wuyts, and P. H. Van de Heyning. Organisation of a universal newborn hearing screening programme in Flanders. B-ENT, 3(4):185-90, Jan. 2007. 